\title{
Adult cervical lymphangioma - ultrasonography, surgical removal, and pathology results. Case report.
}

\author{
Adrian Costache ${ }^{1}$, Mihai Dumitru², Daniel Tweedie ${ }^{3}$, Codrut Sarafoleanu ${ }^{4}$, Ion Anghel ${ }^{2}$
}

${ }^{1}$ Pathology Professor, Ultrasound Teaching Center, Carol Davila University of Medicine and Pharmacy, Bucharest, Romania, ${ }^{2}$ ENT Department, Coltea Clinical Hospital Carol Davila University of Medicine and Pharmacy, Bucharest, Romania, ${ }^{3}$ Guy's and St Thomas' NHS Foundation Trust, London, United Kingdom, ${ }^{4}$ Saint Mary Clinical Hospital, Carol Davila University of Medicine and Pharmacy, Bucharest, Romania

\begin{abstract}
Lymphangioma is a rare pathology in children and even more exotic when encountered in adults. We review the current data on this subject underlining possible controversies and limitations. This is the case of an adult male who underwent a complete clinical examination and ENT performed ultrasound exam, along with contrast CT prior to complete surgical removal of the mass. The mass was $6 \mathrm{~cm}$ in diameter and compressed the left lobe of the thyroid gland. Pathology result confirmed the diagnosis of lymphagioma.
\end{abstract}

Keywords: lymphangioma, ENT, ultrasound, adult

\section{Introduction}

Lymphangioma, known also as cystic hygroma, represents $5 \%$ of the benign congenital malformation arising in childhood and at cervical level represents a sequestration of the embryonic lymphatic channels usually at the level of the posterior cervical triangle [1]. Lymphangiomas are classified microscopically into four categories: lymphangioma simplex (lymphangioma circumscriptum) - small, thin-walled lymphatics; cavernous lymphangioma - dilated lymphatic vessels; cystic lymphangioma (cystic hygroma) - huge, macroscopic lymphatic spaces; and benign acquired progressive lymphangioma in which lymphatic channel dissects through dense collagen bundles [2]. The differential diagnosis includes thyroglossal duct cyst, lipoma, dermoid cyst, laryngocoele, hemangioma, branchial cleft cysts, seromas, metastatic lesions, lymphoma, and ranulas [3]. Two

Received 03.05.2015 Accepted 10.06.2015

Med Ultrason

2015, Vol. 17, No 3, 411-413

Corresponding author: Mihai Dumitru, MD, ENT Resident, $\mathrm{PhD}$ Student,

33/1 Sarandy Frosa street

011231 Bucharest, Romania

Phone: +40721752318

E-mail: orldumitrumihai@yahoo.com thirds of the cases are nowadays diagnosed at birth or in the first 2 years of life, but the prenatal diagnosis using ultrasonography is possible in some cases [4]. The lesion is unilateral, doughy on palpation, and transilluminant [5]. Oral localization is the second most frequent type for lymphangiomas [6] with macroglosia posing great problems for the anesthesiologist [7]. The most useful diagnostic imaging modality is ultrasonography as it enables serial real time analysis of the case without the radiation implied by $\mathrm{CT}$ or the costs added by MRI use [8]. In infancy cervical lymphangiomas are potentially life-threatening due to direct localization or external compression on the airway requiring either a tracheotomy during conservative nonsurgical treatment or a quick surgical removal [9]. However, there are very few cases reported in adults and due to their slow progression there is still a debate concerning their congenital or acquired nature [10]. Treatment options range from sclerosing agents such as bleomycin [11] and OK-432 [12] to radiofrequency ablation [13] ending with the gold standard of surgical excision. Regardless of the treatment options there is a risk of recurrence mostly in suprahyoid lymphangiomas [14].

We present the case of an adult cervical lymphangioma investigated by US and CT and successful surgically removed. 


\section{Case report}

A 38 year old male patient presented to the ENT Department with a long term progressive anterior cervical mass (in the last 10 years) Clinical examination found a $4 \mathrm{~cm}$ tumoral mass situated above the clavicle and extending from the sternocleidomastoid muscle towards the posterior triangle on the left side of the neck. The mass was compressible on palpation creating mild discomfort to the patient (fig 1a). The patient ignored the existence of this mass till recently as it grew progressively in dimensions creating discomfort in wearing clothes and esthetic problems. The rest of the clinical exam and laboratory findings were normal.

Ultrasonography performed using a portable Sonoscape S2 device with a $12 \mathrm{MHz}$ linear probe revealed a supraclavicular complex tumor, oval, poorly delineated from adjacent structures by a thin capsule. The mass had transonic liquid content, multiple septa, dimensions of 46.5/22.3 mm, and no Doppler signal. The structure was situated adjacent to the common carotid artery and internal jugular vein and no regional lymph nodes were found (fig $1 b, c)$. The structure was compressible with the transducer but without being very painful. Taking into consideration all the above mentioned findings our intermediate diagnosis was of a lymphangioma. One important problem was if the mass presented a major extension at the level of the mediastinum thus requiring a combined surgical team with thoracic surgeons.

The ultrasound exam was followed by CT scan in order to ascertain the possible retrosternal extension. Contrast CT exam depicted the contact of the mass with superior vena cava (inferior), and left subclavian vein (medial), the upward extension under the sternocleidomastoid muscle and anterior to the left jugular vein (fig 1d), the thin wall, liquid content, and no enhancement on contrast. The $\mathrm{CT}$ scan confirmed the non invasive nature of the lesion with only compressive properties. Applying the same differential diagnosis the $\mathrm{CT}$ exam reinforced the initial suspicion of lymphangioma.
The chosen treatment for this case was the surgical removal of the mass. The dissection was time consuming as the mass presented very thin walls and contained a yellowish fluid with a high risk of breaking and spilling the content. As shown in figure 1e we removed the entire mass in one piece and the pathology result confirmed our suspicion of lymphangioma. The evolution was favorable with no recurrence in six months follow up.

\section{Discussions}

Lymphangioma is one of the rarest congenital malformations of the neck. Literature states that only $5 \%$ of pediatric benign congenital masses are lymphangiomas and moreover, from these only $8 \%$ are localized at the level of the neck. Our search using PubMed revealed only case presentations on the subject of adult cervical lymphangioma proving that adult patients are very rare, the majority of the cases being solved in infancy. However, our case had also an extension towards the posterior aspect of the sterna manubrium which complicated the surgical removal by the close proximity with the major vessel of the neck. We believe that it was the case of a slowly growing congenital mass neglected by the patient due to poor access to medical facilities and medical education. Ultrasound was very important as it permitted first hand surgeon examination of the mass and preoperative planning. Moreover, by performing the ultrasound exam at the bed side by the ENT surgeon, we had a better communication with the patient. We explained to him the pathology and the ultrasound exam, the patient could visualize in real time the close proximity of this mass with the great vessels of the neck and better understood the risks of the operation. From the point of view of the ultrasound exam, it was similar to other cases described. $\mathrm{CT}$ was performed only for a higher level of accuracy and to evaluate the mediastinal involvement. In the presence of a strictly localized lesion at the level of the neck, ultrasound would have been self sufficient.

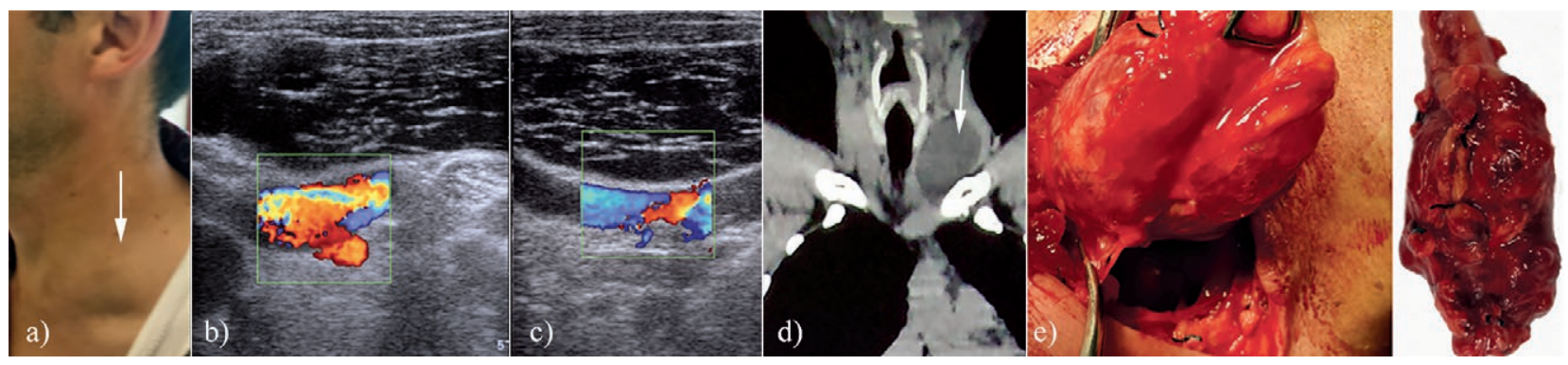

Fig 1. a) Clinical aspect of the patient (arrow- the tumor); b) transverse and c) longitudinal scan ultrasonography demonstrating the relationship with the great vessels; d) Contrast enhanced CT; lymphangioma (arrow) has thin wall, native density between 18 and 34 HU with minimal peripheral enhancement; e) Surgical view of the procedure and the removed specimen. 
In conclusion, the diagnosis of cervical lymphangioma in adults should be taken into consideration at an ultrasonographic examination of a tumor with liquid content and multiple septa.

Acknowledgements: This paper is supported by the Sectoral Operational Programme Human Resources Development (SOP HRD), financed from the European Social Fund and by the Romanian Government under the contract number POSDRU/159/1.5/S/137390.

\section{References}

1. Gaddikeri S, Vattoth S, Gaddikeri RS, et al. Congenital cystic neck masses: embryology and imaging appearances, with clinicopathological correlation. Curr Probl Diagn Radiol 2014; 43: 55-67.

2. Shetty DC, Urs AB, Rai HC, Ahuja N, Manchanda A. Case series on vascular malformation and their review with regard to terminology and categorization. Contemp Clin Dent 2010; 1: 259-262.

3. Davenport M. ABC of general surgery in children. Lumps and swellings of the head and neck. BMJ 1996; 312: 368371.

4. Ghritlaharey RK. Management of giant cystic lymphangioma in an infant. J Clin Diagn Res 2013; 7: 1755-1756.

5. Guruprasad Y, Chauhan DS. Cervical cystic hygroma. J Maxillofac Oral Surg 2012; 11: 333-336.
6. Ganesh C, Sangeetha GS, Narayanan V, Umamaheswari TN. Lymphangioma circumscriptum in an adult: an unusual oral presentation. J Clin Imaging Sci 2013; 3: 44.

7. Bhalla AP, Mehta S, Garg R. Anesthetic management of a child posted for excision of lymphangioma of the tongue. Singapore Med J 2012; 53: e45-e48.

8. Romeo V, Maurea S, Guarino S, et al. A case of lower-neck cystic lymphangioma: correlative US, CT and MR imaging findings. Quant Imaging Med Surg 2013; 3: 224-227.

9. Thompson DM, Kasperbauer JL. Congenital cystic hygroma involving the larynx presenting as an airway emergency. J Nat Med Assoc 1994; 86: 629-632.

10. Stanescu L, Georgescu EF, Simionescu C, Georgescu I. Lymphangioma of the oral cavity. Rom J Morphol Embryol 2006; 47: 373-377.

11. Erikci V, Hosgor M, Yildiz M, et al. Intralesional bleomycin sclerotherapy in childhood lymphangioma. Turk J Pediatr 2013; 55: 396-400.

12. Alonso J, Barbier L, Alvarez J, et al. OK432 (picibanil) efficacy in an adult with cystic cervical lymphangioma. A case report. Med Oral Patol Oral Cir Bucal 2005; 10: 362366.

13. Kim SW, Kauvanough K, Orbach DB, Alomari AI, Mulliken JB, Rahbar R. Long-term outcome of radiofrequency ablation for intraoral microcystic lymphatic malformation. Arch Otolaryngol Head Neck Surg 2011; 137: 1247-1250.

14. Zainine R, El Aoud C, Sellami M, Beltaief N, Sahtout S, Besbes G. Cystic hygroma: report of 25 cases. Tunis Med 2012; 90: 19-24. 